\title{
Towards an explanation of features in the diagnostic diagram of a model atmosphere
}

\section{Linear wave equations with convenient invariants}

\author{
F. Schmitz ${ }^{1}$ and B. Fleck ${ }^{2}$ \\ ${ }^{1}$ Institut für Theoretische Physik und Astrophysik der Universität Würzburg, Am Hubland, 97074 Würzburg, Germany \\ ${ }^{2}$ ESA Research and Scientific Support Department, NASA/GSFC, Mailcode 682.3, Greenbelt, MD 20771, USA
}

Received 4 November 2002 / Accepted 6 December 2002

\begin{abstract}
New standard forms of the time-independent linear adiabatic wave equation of plane atmospheres are presented. The main objective is to obtain equations with invariants as simple as possible so that oscillation theorems can be applied effectively. By transformations of both the independent and the dependent variables, equations with simple invariants are formulated. We present a standard form of the wave equation the invariant of which depends only on the first derivative of the equilibrium density, as opposed to the common standard form the invariant of which depends also on second derivatives. Further, we discuss a procedure which replaces the wave equation by a system of two simple second order differential equations. In this case we try to draw conclusions on the general behavior of solutions by use of oscillation theorems. In addition, a re-formulation of the wave equation is presented, which eliminates terms with first derivatives of atmospheric quantities. The independent variable of the resulting equation depends not only on the geometrical height but also on the ratio $\omega / k$. In this case, it is necessary to use a diagnostic diagram the axes of which are given by $\omega / k$ and $\omega$ instead of the common $k-\omega$ diagram. Therefore we discuss the meaning of the parameter $\omega / k$ for the representation of dispersion curves. Finally, for the VAL-atmosphere (Vernazza et al. 1981), regions of certainly nonoscillatory waves are considered.
\end{abstract}

Key words. Sun: atmosphere - Sun: oscillations - stars: atmospheres

\section{Introduction}

The present paper is a continuation of a paper by Schmitz \& Fleck (1998), called Paper I, which considered vertically propagating adiabatic waves in a plane atmosphere. There, common standard forms of the 1-dimensional wave equation are discussed critically and a wave equation with a very simple invariant is presented. We now have performed extensions of the strategy of Paper I to the three-dimensional propagation of linear adiabatic waves in plane atmospheres. Here, the problem of the reformulation of the time-independent wave equation is significantly more complex than in the one-dimensional case.

To solve the wave equation numerically, one takes a corresponding system of two first order equations. In general, one uses the two linearized hydrodynamical equations from which the wave equation is formed. If we take appropriate dependent variables (vertical displacement and Lagrangian pressure perturbation), then this system is uncomplicated, and can easily be integrated numerically.

Send offprint requests to: F. Schmitz
To obtain solutions in closed form for simple analytical model atmospheres, the representation of the wave equation and the choice of the variables are crucial. In that case one tries to transform the wave equation to known second order equations, in particular equations of special functions (e.g. Schmitz \& Steffens 1999).

To understand the behavior of waves without solving the wave equation explicitely or to interprete results of numerical calculations, one writes the wave equation in standard form and studies the behavior of the invariant. Besides, the standard form is also used for a numerical integration, as there are particular numerical integration methods for so-called Schoedingertype equations. It is clear that a simple invariant is convenient. Further, a simple invariant is useful in connection with approximation methods of solutions as the WKB-approximation (e.g. Mathews \& Walker 1970).

Recent investigations of resonances of standing waves in the VAL-atmosphere and modifications (cool chromosphere or shifted transition layer) of this atmosphere by Steffens et al. (1997) have yielded a wealth of features in the diagnostic diagram. Further, investigations of the influence of atmospheric 
layers (VAL-atmosphere and modifications) on the p-modes of the sun by Steffens (1998), and Steffens \& Schmitz (2000) have led to complicated dispersion curves. The corresponding ridges in the diagnostic diagram show strong bending or indicate avoided crossing. For the VAL-atmosphere, the frequency range where such effects occur is $3<v<7 \mathrm{mHz}$. The results are not yet interpreted and understood.

A frequently discussed, but not commonly accepted phenomenon is that of a chromospheric mode, presumably due to an acoustic cavity between the temperature minimum and the transition region (cf. e.g. Leibacher \& Stein 1981). For a detailed discussion refer to Steffens et al. (1985), who report on signatures of a chromospheric mode at $6 \mathrm{mHz}$ in their observations. However, Steffens \& Schmitz (2000) demonstrated that the corresponding features can appear in a $k-\omega$ diagram even without a chromosphere, i.e. without a temperature minimum and a chromospheric temperature increase. It therefore does not necessarily require invoking a chromospheric mode to explain these features. Further, their results show that, in order to understand the diagnostic diagram of the Sun, it is useful to consider modifications and variations of the outer layers. It remains open, however, how to best explain certain features in diagnostic diagrams - by physical systems (e.g. cavities) or by a discussion of the equations. A nice example can be found in Aizenman et al. (1977). They have investigated the behavior of $l=2$-modes of stars "evolving away from the main sequence". There, in a frequency-age diagram mode bending (bumping) similar to that in the solar $\omega-k$ diagram occurs. The authors have shown that the bending of the ridges can be explained by avoided crossing of the ridges of two single systems. These systems however, are fictitious systems defined by equations which are obtained by simplifying the full equations.

However that may be, the common standard form of the adiabatic wave equation is not suitable to explain and understand the features. Further, it is not clear which combinations of the wave number $k$ and the frequency $\omega$ should be taken to understand the behavior of structures and ridges in the diagnostic diagram: the pairs $(k, \omega)$ or $(\omega / k, \omega)$ or $\left(k, \omega^{2} / k\right)$ etc.?

Paper I was closed by the presentation of a simple standard form of the equation of vertically propagating waves. In the case of the VAL-atmosphere, the numerical behavior of the invariant of this equation is very simple. The three-dimensional case is much more complicated and the optimal standard form of the equation is not unique. Thus, in the present paper we concentrate on the mathematical tools. A subsequent paper by co-workers will deal with features and ridges in diagnostic diagrams.

The present paper is organized as follows: in Sect. 2 we present the linearized hydrodynamic equations and the resulting wave equation. Section 3 deals with the common standard form of the wave equation. This form is critized in Sect. 4 where also oscillation theorems are given. In Sect. 5, we present and discuss some new standard forms of the wave equations. The equation given in Sect. 5.1 is obtained by introducing the logarithmic mass as the independent variable. In Sect. 5.2 we separate the wave equation into two second order differential equations and apply oscillation theorems to obtain general statements on the behavior of solutions. A standard form of the wave equation the invariant of which is free of first derivatives of atmospheric quantities is presented in Sect. 6. In Sect. 7 we discuss criteria for nonoscillatory waves with applications to the VAL-atmosphere.

\section{Notations and the original wave equations}

Let $z$ be the vertical, outwards directed geometrical coordinate and $g$ the constant gravity. Let $p(z)$ and $\rho(z)$ be the pressure and the density of the equilibrium atmosphere, $c(z)$ the adiabatic sound speed, $\xi(\boldsymbol{r}, t)$ the vertical component of the Lagrangian displacement and $\Delta p(\boldsymbol{r}, t)$ the Lagrangian pressure perturbation. The horizontal position $\boldsymbol{r}_{\|}$and the time $t$ are separated by $\exp \left[i\left(\omega t-\boldsymbol{k} \boldsymbol{r}_{\|}\right)\right]$, where $\omega$ is the real frequency, and $\boldsymbol{k}$ the horizontal wave vector.

From the linearized hydrodynamic equations we obtain two first order differential equations (cf. e.g. Schmitz \& Fleck 1994)

$$
\begin{aligned}
& \frac{\mathrm{d}}{\mathrm{d} z} \xi-g \frac{k^{2}}{\omega^{2}} \xi=\frac{1}{\omega^{2} \rho c^{2}}\left(c^{2} k^{2}-\omega^{2}\right) \Delta p \\
& \frac{\mathrm{d}}{\mathrm{d} z} \Delta p+g \frac{k^{2}}{\omega^{2}} \Delta p=\rho\left[\omega^{2}-\frac{k^{2} g^{2}}{\omega^{2}}\right] \xi
\end{aligned}
$$

where now $\xi=\xi(z), \Delta p=\Delta p(z)$, and $k=|\boldsymbol{k}|$. We use the variables $\xi$ and $\Delta p$ as only these perturbations are continuous at a contact discontinuity where $\rho$ and $c$ are discontinuous. Because of the continuity of $\xi$ and $\Delta p$, the coefficients of the equations cannot contain derivatives of $\rho$ and $c$. In the case of other variables as the Eulerian pressure perturbation or the Lagrangian density perturbation the coefficients of the corresponding equations contain first order derivatives of atmospheric quantities like $c$ and $\rho$.

From Eqs. (1) and (2) we obtain the time-independent wave equation of the Lagrangian pressure perturbation $\Delta p$ :

$$
\begin{aligned}
\omega^{2} c^{2} & {\left[\frac{\mathrm{d}^{2} \Delta p}{\mathrm{~d} z^{2}}-\frac{1}{\rho} \frac{\mathrm{d} \rho}{\mathrm{d} z} \frac{\mathrm{d} \Delta p}{\mathrm{~d} z}\right] } \\
- & -\left[k^{2} g\left(g+\frac{c^{2}}{\rho} \frac{\mathrm{d} \rho}{\mathrm{d} z}\right)+\omega^{2}\left(c^{2} k^{2}-\omega^{2}\right)\right] \Delta p=0 .
\end{aligned}
$$

The Lagrangian pressure perturbation $\Delta p$ is a scalar, the vertical displacement $\xi$ a component of a vector. The corresponding wave equation of $\xi$ has a critical level at $\omega=c(z) k$. Hence, it is more complicated than the equation of $\Delta p$.

\section{The common standard form of the wave equation}

For this form (see e.g. Deubner \& Gough 1984), the independent variable is the geometrical height $z$. With the transformation

$\Psi(z)=\rho^{-1 / 2} \Delta p(z)$

the wave Eq. (3) turns into the standard form

$\frac{\mathrm{d}^{2}}{\mathrm{~d} z^{2}} \Psi+Q(z) \Psi=0$ 
where the invariant $Q(z)$ is given by

$$
Q(z)=\frac{1}{c^{2}}\left(\omega^{2}-\omega_{0}^{2}\right)+k^{2}\left(\frac{N^{2}}{\omega^{2}}-1\right)
$$

with

$\omega_{0}^{2}=\frac{c^{2}}{4}\left[\frac{3}{\rho^{2}}\left(\frac{\mathrm{d} \rho}{\mathrm{d} z}\right)^{2}-\frac{2}{\rho} \frac{\mathrm{d}^{2} \rho}{\mathrm{d} z^{2}}\right]$

and

$N^{2}=g\left[-\frac{1}{\rho} \frac{\mathrm{d} \rho}{\mathrm{d} z}-\frac{g}{c^{2}}\right]$.

In the case of the isothermal atmosphere, $\omega_{0}$ is the constant acoustic cut-off frequency and $N$ the constant Brunt-Vaisala frequency. The factorized form of the invariant $Q(z)$ reads:

$Q(z)=\frac{\omega^{2}}{c^{2}}\left(1-\frac{\omega_{+}^{2}}{\omega^{2}}\right)\left(1-\frac{\omega_{-}^{2}}{\omega^{2}}\right)$

where

$\omega_{ \pm}^{2}=\frac{1}{2}\left(k^{2} c^{2}+\omega_{0}^{2}\right) \pm \sqrt{\frac{1}{4}\left(k^{2} c^{2}+\omega_{0}^{2}\right)^{2}-N^{2} k^{2} c^{2}}$

(cf. Deubner \& Gough 1984).

The standard form given here is the most common. Occasionally, other forms of the wave equation are used. Mihalas \& Toomre (1981) used a standard form which was obtained by the wave equation of the vertical displacement $\xi$.

\section{Critique of the standard form and oscillation theorems}

The common standard form of the wave equation is not suitable, as it requires the calculation of the second derivative of the density, which is nearly impossible to do in the case of an empirical atmosphere.

The invariant used by Mihalas \& Toomre (1981) contains the second derivative of the adiabatic sound speed (comparable to the second derivative of the density in Eq. (7)). The authors write: "however, it was found to be essential to smooth the model in order to obtain continuos derivatives of the sound speed and density scale height. Otherwise small scale fluctuations in the vertical wave number produce numerous partial reflections which are artifacts of the model and have no physical basis". Numerical differentiation is usually a difficult operation, particularly in the case of empirical data. In Paper I we calculated the second derivative of the adiabatic sound speed of the VAL-atmosphere. There, small deviations from the VAL-data strongly influenced the second derivative.

Besides, the numerical behavior of the invariant is far too complicated to draw conclusions to the form of the solutions. In Paper I we have extensively discussed this problem for the vertical case $k=0$. The so-called invariant of a second order differential equation is not a physical invariant, i.e. a scalar field, but it changes when the variables are transformed. In Paper I we have compared some different representations of the wave equation. We have seen how the invariants undergo strong changes when the variables are transformed. It was shown that the behavior of waves can be described by a wave equation, the invariant of which contains only one coefficient, which is the quantity $p / \rho c$. In the VAL atmosphere, and there in particular in the chromosphere, the behavior of this quantity differs markedly from the behavior of the temperature or the adiabatic sound speed.

An uncomplicated and computationally simple invariant plays a role also in the following case: for a numerical integration, a differential equation of higher order is replaced by a system of first order equations. There is an exception to this rule. For the numerical integration of second order differential equations whithout the first derivative, there are particular methods. Mihalas \& Toomre (1981) have used such a method to solve the wave equation.

Oscillation and nonoscillation theorems for second order equations are given in Gradshteyn \& Ryzhik (1980). Only a few oscillation theorems for the solutions of second order differential equations are really useful. Most of such theorems concern infinite intervals. (Mathematically, solutions are said to be oscillatory if they possess an infinite number of zeros in the interval $[a, \infty)$, nonoscillatory, if they possess only a finite number of zeros.)

For this paper, we need only the following two nonoscillating theorems for a differential equation $y^{\prime \prime}+f(x) y=0$ :

1. If $f \leq 0$ on an interval $(a, b)$ or on $(-\infty,+\infty)$, then $y(x)$ has not more than one zero in this interval. In this paper, we call this case nonoscillatory.

2. There are strictly increasing and decreasing linearly independent positive solutions if $f<0$ is continuous in $(-\infty,+\infty)$ and if $x f(x)$ is not integrable on $(0, \infty)$. This property corresponds to the existence of the solutions $\mathrm{e}^{-x}$ and $\mathrm{e}^{+x}$ of the equation $y^{\prime \prime}-y=0$ where only these solutions, apart of constant factors, are strictly monotonous and positive as opposed to the solutions $\sinh x$ or $\cosh x$. As we shall consider atmospheric layers with finite extension the integrability of the function $x f(x)$ is unimportant. For $f<0$ we can always find a monotonic and positive solution.

When the invariant is negative, the solutions are nonoscillatory. This statement is sufficient, but not necessary. So, $f(x)=0$ does not separate exponential and oscillatory behavior as it is often claimed. Only criteria for certainly nonoscillatory behavior can be given.

\section{Different forms of the wave equation}

Using the column mass $m$ given by

$m=\int_{z}^{\infty} \rho(z) \mathrm{d} z \quad$ or $\quad \mathrm{d} m=-\rho \mathrm{d} z$,

we obtain:

$\frac{\mathrm{d}^{2} \Delta p}{\mathrm{~d} z^{2}}-\frac{1}{\rho} \frac{\mathrm{d} \rho}{\mathrm{d} z} \frac{\mathrm{d} \Delta p}{\mathrm{~d} z}=\rho^{2} \frac{\mathrm{d}^{2} \Delta p}{\mathrm{~d} m^{2}}$.

Then, the wave Eq. (3) reads:

$\frac{\mathrm{d}^{2} \Delta p}{\mathrm{~d} m^{2}}+\frac{1}{\omega^{2} c^{2} \rho^{2}}\left[k^{2} g\left(c^{2} \frac{\mathrm{d} \rho}{\mathrm{d} m}-g\right)+\omega^{2}\left(\omega^{2}-c^{2} k^{2}\right)\right] \Delta p=0$. 
For

$k^{2} g\left(c^{2} \frac{\mathrm{d} \rho}{\mathrm{d} m}-g\right)+\omega^{2}\left(\omega^{2}-c^{2} k^{2}\right)<0$

solutions are certainly nonoscillatory. This condition, however, is weak: in the case of an isentropic stratification it reduces to $\omega<c k$, in the case $k=0$ to $\omega^{2}<0$.

It is not of advantage to replace the density $\rho$ by the temperature $T$, as $\rho(m)$ increases monotonously, whereas $T(m)$ is not monotonous. Because of the Schwarzschild criterion for convective stability,

$\left.\frac{\mathrm{d} p}{\mathrm{~d} \rho}\right|_{\text {star }}<\left.\frac{\mathrm{d} p}{\mathrm{~d} \rho}\right|_{\text {adiabatic }}=c^{2}$

and because of $p=m g$, we have

$c^{2} \frac{\mathrm{d} \rho}{\mathrm{d} m}-g>0$

so that the corresponding term of the invariant of the wave Eq. (13) is positive. For $k=0$, the wave equation reduces to:

$\frac{\mathrm{d}^{2}}{\mathrm{~d} m^{2}} \Delta p+\frac{\omega^{2}}{c^{2} \rho^{2}} \Delta p=0$

As regards the representation of the propagation characteristics of a wave, the mass is a somewhat disadvantegeous coordinate. A wave equation which in the case of the isothermal atmosphere reduces to a differential equation with constant coefficients is more familiar. So instead of the mass we should use a geometrical coordinate. First, however, we present a more general form of the wave equation.

We define a new independent variable $x$ by

$\mathrm{d} m=-u^{2}(x) \mathrm{d} x$

with arbitrary $u(x)$. The coordinate $x$ is outwards directed. By this transformation, we obtain an equation which contains the first derivative of $\Delta p$. To eliminate this derivative, we have to put

$\Delta p=u(x) v(x)$

with $v(x)$ as a new dependent variable. We obtain

$\frac{\mathrm{d}^{2} \Delta p}{\mathrm{~d} m^{2}}=\frac{1}{u^{3}} \frac{\mathrm{d}^{2} v}{\mathrm{~d} x^{2}}+\frac{\mathrm{d}^{2} u}{\mathrm{~d} m^{2}} v$

so that the wave Eq. (13) takes the form

$$
\begin{aligned}
\frac{\mathrm{d}^{2} v}{\mathrm{~d} x^{2}}+v u^{3} & \frac{\mathrm{d}^{2} u}{\mathrm{~d} m^{2}}+\frac{u^{4}}{\omega^{2} c^{2} \rho^{2}} \\
& \times\left[k^{2} g\left(c^{2} \frac{\mathrm{d} \rho}{\mathrm{d} m}-g\right)+\omega^{2}\left(\omega^{2}-c^{2} k^{2}\right)\right] v=0 .
\end{aligned}
$$

Here, the function $u(m)$ is arbitrary. In the following sections we try to choose the function $u(m)$ so that the differential equation simplifies. We discuss two possibilities: we set $u(m)$ or we define $u(m)$ by a linear second order differential equation.

\subsection{Introduction of a quasi-geometrical coordinate}

We proceed as in the case $k=0$ in Paper I. Let $H$ be an arbitrary scale height. Putting

$u(m)=\sqrt{m / H}$,

we obtain

$u^{3} \frac{\mathrm{d}^{2} u}{\mathrm{~d} m^{2}}=-\frac{1}{4 H^{2}}$.

Further, we get

$\mathrm{d} x=-H \frac{\mathrm{d} m}{m} \quad$ or $\quad x=-H \log \left(\frac{m}{m_{0}}\right)$,

so that the coordinate $x$ has the dimension of a length. Finally, we obtain the equation

$$
\begin{aligned}
\frac{\mathrm{d}^{2} v}{\mathrm{~d} x^{2}}+ & \frac{1}{H^{2}}\left[\frac{p^{2}}{g^{2} \omega^{2} c^{2} \rho^{2}}\right. \\
& \left.\left(k^{2} g\left(c^{2} \frac{\mathrm{d} \rho}{\mathrm{d} m}-g\right)+\omega^{2}\left(\omega^{2}-c^{2} k^{2}\right)\right)-\frac{1}{4}\right] v=0,
\end{aligned}
$$

where we have put $m=p / g$. For $k=0$, this equation reduces to the equation recommended in Paper I as the basic equation of vertically propagating waves:

$\frac{\mathrm{d}^{2} v}{\mathrm{~d} x^{2}}+\frac{1}{H^{2}}\left[\frac{\omega^{2} p^{2}}{g^{2} c^{2} \rho^{2}}-\frac{1}{4}\right] v=0$.

By Eqs. (24), we have introduced a quasi-geometrical coordinate $x$. The dependent variable becomes $v=\Delta p / \sqrt{p}$. For constant adiabatic sound speed $c$, Eq. (25) reduces to the usual wave equation of the isothermal atmosphere and $x$ becomes the real geometrical coordinate $z$. As $p=m g$ and $m=m_{0} \exp (-x / H)$ the pressure perturbation is given by

$\Delta p(x)=\exp (-x / 2 H) v(x)$.

When

$\omega^{4}-c^{2} k^{2} \omega^{2}-\frac{1}{4} \frac{g^{2} \omega^{2} c^{2} \rho^{2}}{p^{2}}+k^{2} g\left(c^{2} \frac{\mathrm{d} \rho}{\mathrm{d} m}-g\right)<0$,

the solutions $v(x)$ of Eq. (25) are certainly nonoscillatory.

\subsection{Definition of the function $u(m)$ by a differential equation}

We put

$\frac{\mathrm{d}^{2} u}{\mathrm{~d} m^{2}}-F(m) u=0$

where $F(m)$ must be a positive function which is composed of parts of the third term of Eq. (21). Then, the solutions $u(m)$ of Eq. (29) are nonoscillatory functions. Corresponding to theorem 2 of Sect. 4 there exists a monotonous positive solution. Let $u_{0}(m)$ be such a solution. Then, integration of

$\frac{\mathrm{d} x}{\mathrm{~d} m}=-\frac{1}{u_{0}^{2}(m)}$ 
yields a monotonous function $x(m)$. Finally, we obtain a monotonous positive function $u_{0}(x)$. As the function $F(m)$ contains parts of the third term of Eq. (21), this equation simplifies.

We consider only two cases. First, we put

$\frac{\mathrm{d}^{2} u}{\mathrm{~d} m^{2}}-\frac{1}{c^{2} \rho^{2}}\left(\frac{k^{2} g^{2}}{\omega^{2}}-\omega^{2}\right) u=0$.

In this case, the solutions $u(m)$ are certainly nonoscillatory only for $\omega^{2}<g k$ so that this case applies to gravity waves. Let $u_{0}(m)$ be a positive monotonous solution. Then, also $u_{0}(x)$ is positive and monotonous. The wave Eq. (21) reduces to:

$\frac{\mathrm{d}^{2} v}{\mathrm{~d} x^{2}}+\frac{u_{0}^{4} k^{2}}{\rho^{2} \omega^{2}}\left[g \frac{\mathrm{d} \rho}{\mathrm{d} m}-\omega^{2}\right] v=0$.

The invariant of this equation is negative for $g \frac{\mathrm{d} \rho}{\mathrm{d} m}<\omega^{2}$, and hence, the solutions $v(x)$ are certainly nonoscillatory if

$k g>\omega^{2}>g \frac{\mathrm{d} \rho}{\mathrm{d} m}$.

This condition holds for extremely high values of $k$, and is therefore unimportant. For $c \rightarrow \infty$, a trivial solution of Eq. (31) is $u=1$. For $u=u_{0}=1$, Eq. (32) becomes

$\frac{\mathrm{d}^{2} v}{\mathrm{~d} m^{2}}+\frac{k^{2}}{\rho^{2} \omega^{2}}\left[g \frac{\mathrm{d} \rho}{\mathrm{d} m}-\omega^{2}\right] v=0 \quad$ with $\quad v=\Delta p$.

This is the wave equation of the so-called "heterogeneous incompressible model" (Ledoux \& Walraven 1958) in the plane layer approximation. By this model, gravity waves are described approximately, acoustic waves are suppressed. Hence, the function $u_{0}(x)$ describes deviations of incompressibility. It is evident, that the form of the invariant of Eq. (32) is relativly simple. Hence, for gravity waves with $\omega^{2}<g k$ no particular effects should occur. In fact, the ridges of gravity modes of the sun (Cowling's and plane layer approximation, Steffens \& Schmitz 2000) show no particular features.

More important than gravity waves are acoustic waves. In this case, we put

$\frac{\mathrm{d}^{2} u}{\mathrm{~d} m^{2}}+\frac{k^{2}}{\rho^{2} \omega^{2}}\left[g \frac{\mathrm{d} \rho}{\mathrm{d} m}-\omega^{2}\right] u=0$.

The solutions $u(m)$ of this equation are nonoscillatory for

$\omega^{2}>g \frac{\mathrm{d} \rho}{\mathrm{d} m}$.

From the maximum of $\mathrm{d} \rho / \mathrm{d} m$ shown in Fig. 1 we can infer that this condition is fulfilled for frequencies $v>9 \mathrm{mHz}$, i.e. above the range $3<v<7 \mathrm{mHz}$, mentioned in the introduction. There is a monotonic solution $u_{0}(m)$ and hence a function $u_{0}(x)$, which is monotonic and positive. For $v(x)$ we obtain the equation

$\frac{\mathrm{d}^{2} v}{\mathrm{~d} x^{2}}+\frac{u_{0}^{4}}{c^{2} \rho^{2}}\left(\omega^{2}-\frac{k^{2} g^{2}}{\omega^{2}}\right) v=0$.

The solution $v(x)$ is certainly nonoscillatory for $\omega^{2}<g k$, so that we again obtain the condition (33). The invariant of Eq. (37) is simple. For sound waves where $\omega^{2}>k g$ it does not change the sign. Hence we may infer that no pecularities will occur for frequencies $v>9 \mathrm{mHz}$. Results of the investigations of Steffens \& Schmitz (2000) and of Steffens et al. (1997) confirm this conclusion.

Therefore, for a given atmospheric stratification, only waves with frequencies

$g \frac{\mathrm{d} \rho}{\mathrm{d} m}>\omega^{2}>k g$

should show striking features. In this frequency range, it is not possible to separate Eq. (21) conveniently. Thus, to discuss acoustic waves with $3<v<7 \mathrm{mHz}$ we have to use Eq. (25). However, there is another possibility.

\section{A further representation of the wave equation}

In the following we combine Eqs. (1) und (2) to a wave equation the invariant of which does not contain the derivative of the density. As the coefficients of these equations are free of derivatives, such a procedure should be possible. We found that only one way is practicable. We may assume that $0<z<\infty$. By the transformations of the displacement and the pressure perturbation

$\xi(z)=\mathrm{e}^{g(k / \omega)^{2} z} u(z)$

$\Delta p(z)=\mathrm{e}^{-g(k / \omega)^{2} z} v(z)$

we obtain the equations

$\frac{\mathrm{d} u}{\mathrm{~d} z}=\frac{1}{\omega^{2} \rho c^{2}}\left(k^{2} c^{2}-\omega^{2}\right) \mathrm{e}^{-2 g(k / \omega)^{2} z} v$,

$\frac{\mathrm{d} v}{\mathrm{~d} z}=\left(\omega^{2}-\frac{k^{2} g^{2}}{\omega^{2}}\right) \rho(z) \mathrm{e}^{2 g(k / \omega)^{2} z} u$,

for continuous functions $u(z)$ und $v(z)$. Now we introduce a new independent variable $y$ by

$y=\int_{0}^{z} \rho(z) \mathrm{e}^{2 g(k / \omega)^{2} z} \mathrm{~d} z$.

The ratio $k / \omega$ is assumed to be given. The physical dimension of the variable $y$ is that of a column mass. As the integrand is positiv, the function $y(z)$ increases monotonously with $z$. Therefore, also the inverse $z(y)$ is a monotonous function. The above differential equations read:

$\frac{\mathrm{d} u}{\mathrm{~d} y}=\frac{1}{\omega^{2} \rho^{2} c^{2}}\left(k^{2} c^{2}-\omega^{2}\right) \mathrm{e}^{-4 g(k / \omega)^{2} z} v$,

$\frac{\mathrm{d} v}{\mathrm{~d} y}=\left(\omega^{2}-\frac{k^{2} g^{2}}{\omega^{2}}\right) u$.

Eliminating $u(y)$, we obtain the second-order equation

$\frac{\mathrm{d}^{2} v}{\mathrm{~d} y^{2}}+I(y) v=0$

with the invariant

$I(y)=\left(\omega^{4}-k^{2} g^{2}\right)\left(\omega^{2}-k^{2} c^{2}\right) \frac{\mathrm{e}^{-4 g(k / \omega)^{2} z}}{\omega^{4} \rho^{2} c^{2}}$, 
where $z=z(y)$. The function $z(y)$ can be obtained by a differential equation which corresponds to the integral (43). For

$\sqrt{k g}>\omega>c k$ or $\quad c k>\omega>\sqrt{k g}$

solutions are certainly nonoscillatory.

In the vertical case with $k=0$, the integral (43) reduces to

$y=\int_{0}^{z} \rho(z) \mathrm{d} z=M-m$

where $M$ is the total mass of the layer and $m$ the complement (11). Then, the invariant is $I=\omega^{2} / \rho^{2} c^{2}$, so that Eq. (46) reduces to Eq. (17) if $k=0$. We shall now transform Eq. (46) by introducing a new independent variable. The strategy is to obtain Eq. (26) when $k=0$ and, in the limit of a constant sound speed, the wave equation of the isothermal atmosphere.

First, we introduce a new dependent variable $w$ and an new coordinate $x$ by

$v=w \sqrt{\eta}$

and

$\mathrm{d} y=\eta(x) \mathrm{d} x$.

With these transformations, Eq. (46) reads:

$\frac{\mathrm{d}^{2} w}{\mathrm{~d} x^{2}}+J(x) w+I(x) \eta^{2}(x) w=0$

with

$J(x)=\frac{1}{2} \frac{1}{\eta} \frac{\mathrm{d}^{2} \eta}{\mathrm{d} x^{2}}-\frac{3}{4} \frac{1}{\eta^{2}}\left(\frac{\mathrm{d} \eta}{\mathrm{d} x}\right)^{2}$.

In principle, the form of $\eta(x)$ is free. However, $J(x)$ should be a simple function. Now, if $\eta(x)$ is an exponential, $J$ becomes constant. For this reason, we put

$\eta(x)=\rho_{0} \mathrm{e}^{\kappa x}$

where $\rho_{0}$ is a constant density and

$\kappa=2 g\left(\frac{k}{\omega}\right)^{2}-\frac{1}{H}$.

Then, we have

$\mathrm{d} y=\rho_{0} \mathrm{e}^{\kappa x} \mathrm{~d} x$

We obtain

$J=-\frac{1}{4} \kappa^{2}$

Finally, Eq. (52) reduces to

$\frac{\mathrm{d}^{2} w}{\mathrm{~d} x^{2}}+Q(x) w=0$ with the invariant

$$
\begin{aligned}
Q(x)= & \frac{\rho_{0}^{2}}{\rho^{2} c^{2}}\left(\omega^{2}-\frac{k^{2}}{\omega^{2}} g^{2}\right)\left(1-\frac{k^{2}}{\omega^{2}} c^{2}\right) . \\
& \mathrm{e}^{-2 x / H+4 g(k / \omega)^{2}(x-z)}-\frac{1}{4} \kappa^{2}
\end{aligned}
$$

with $\rho(x), c(x)$, and $z(x)$. The independent variable $x$ has the physical dimension of a length. Hence, it is a quasi-geometrical coordinate. We can obtain the function $z(x)$ by the numerical integration of the simple and uncomplicated differential equation

$\frac{\mathrm{d} z}{\mathrm{~d} x}=\frac{\rho_{0}}{\rho(z)} \mathrm{e}^{-2 g(k / \omega)^{2} z} \mathrm{e}^{K x}$.

It depends on the ratio $k / \omega$.

In the case of the isothermal atmosphere we have:

$x=z, \rho(z)=\rho_{0} \mathrm{e}^{-z / H}, c(z)=c_{0}, H=\frac{\gamma g}{c_{0}^{2}}$,

where $\gamma$ is the adiabatic exponent. In this case, the invariant (59) becomes constant:

$Q=\frac{\left(\omega^{4}-k^{2} g^{2}\right)}{\omega^{4} c_{0}^{2}}\left(\omega^{2}-k^{2} c_{0}^{2}\right)-\left(\frac{1}{2 H}-g \frac{k^{2}}{\omega^{2}}\right)^{2}$.

This invariant yields the familiar dispersion relation of the isothermal atmosphere.

Finally, for $k=0$ the wave equation reduces to Eq. (26), the equation recommended in Paper I. The pressure perturbation is given by

$\Delta p(x)=\mathrm{e}^{-x / 2 H+g(k / \omega)^{2}(x-z)} w(x)$.

Usually, corresponding to the axes $k$ and $\omega$ of the familiar diagnostic diagram, one discusses how solutions of the wave equation depend on the frequency $\omega$ for given $k$. In the case of the wave Eq. (58), however, the parameter $\omega / k$ takes the place of $k$. This is a little inconvenient. However, $\omega / k$ is a physical quantity, the phase velocity. Further, for the ridges of solar p-modes, Duvall (1982) found empirically, that $1 / \omega$ is a simple function of $\omega / k$ alone (Duvall's law, see also Deubner \& Gough 1984, Fig. 3). Also the dispersion curves of the modes of a polytropic layer (a convection zone without an overlaying atmosphere) can be written in the form (Lamb 1932):

$\frac{1}{\omega}=\frac{\omega}{k} \frac{1}{g} f(n, \gamma, j)$

where $f$ is an algebraic function of the polytropic index $n$, the adiabatic exponent $\gamma$, and the vertical wave number $j=$ $0,1,2, \ldots$

Schmitz \& Steffens (1999) have calculated the modes of an asymptotically polytropic convection zone with a smoothly matched, asymptotically isothermal atmosphere. In the case of an asymptotically isentropic stratification of the convection zone (i.e. $\gamma=1+1 / n$ ) the frequency of the $p_{1}$-mode is given by a quadratic equation for $\omega^{2}$ :

$\omega^{4}-(2 \gamma-1) g k \omega^{2}+4(\gamma-1) c_{0}^{2} g k^{3}=0$, 


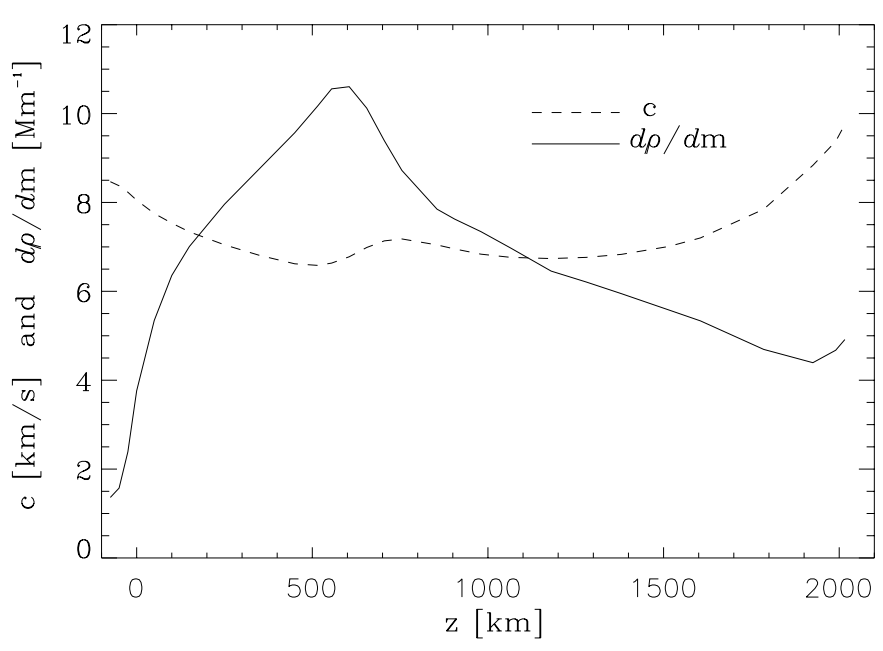

Fig. 1. The adiabatic sound speed and the derivative of the density as functions of the height $z$.

where $c_{0}$ is the asymptotic, adiabatic sound speed of the atmosphere. We can reduce this equation to the simple form

$\frac{1}{\omega}=\left(\frac{\omega}{k}\right)^{3}\left[\left(\frac{\omega}{k}\right)^{2} g(2 \gamma-1)-4(\gamma-1) c_{0}^{2} g\right]^{-1}$.

Also for the other p-modes, the relation $\omega(\omega / k)$ is simpler than the relation $\omega(k)$. These findings are in agreement with Duvall's law. We thus think that the use of $\omega / k$ instead of $k$ as a parameter of the invariant $Q(x)$ and the geometrical coordinate $z(x)$ makes sense.

\section{Regions of certainly nonoscillatory waves}

We now give inequalities which hold for realistic frequencies and wave numbers. In regions, where these inequalities hold, waves are certainly nonoscillatory. In Sect. 6, we obtained:

$\sqrt{k g}>\omega>c k$ or $c k>\omega>\sqrt{k g}$.

These conditions, however, are weak as they define only small regions of the diagnostic diagram. The condition given in Sect. 5.1 is more important:

$\omega^{4}-\omega^{2}\left(c^{2} k^{2}+\omega_{0}^{2}\right)+k^{2} g\left(c^{2} \frac{\mathrm{d} \rho}{\mathrm{d} m}-g\right)<0$.

with

$\omega_{0}=\frac{g c \rho}{2 p}$.

The curves $\omega(k, z)$ given by the square-roots of $\omega^{2}=$

$\frac{1}{2}\left[\left(c^{2} k^{2}+\omega_{0}^{2}\right) \pm\left[\left(c^{2} k^{2}+\omega_{0}^{2}\right)^{2}-4 k^{2} g\left(c^{2} \frac{\mathrm{d} \rho}{\mathrm{d} m}-g\right)\right]^{1 / 2}\right]$

define regions with certainly nonoscillatory waves.

Let us now consider the VAL-atmosphere (Vernazza et al. 1981). We take the temperature stratification $T(z)$ and the pressure $p(z)$ of this atmosphere. We calculate the density $\rho(z)$ and the adiabatic sound speed $c(z)$ including dissociation and ionization of hydrogen and ionization of helium: $\mathrm{H}_{2}, \mathrm{H}, \mathrm{H}^{+}, \mathrm{He}$, $\mathrm{He}^{+}, \mathrm{He}^{++}, \mathrm{e}^{-}$. We have used the LTE-code described by Wolf (1983). The calculated densities coincide with the tabulated densities of the VAL-atmosphere. Only in the upper chromosphere $(z>1500 \mathrm{~km})$ there are deviations, because of the NLTE-effects of the VAL-atmosphere. We therefore may assume that also the calculated adiabatic sound speed is correct. In the paper of Steffens \& Schmitz (2000), the VAL- atmosphere was matched to the convection zone of Spruit (1977). There an equidistant data set was generated from the tabulated points of the convection zone and the VAL-atmosphere. This set, which was used to interpolate the coefficients of Eqs. (1) and (2) for the numerical integration, is not suitable to calculate the derivative of the density. We have therefore calculated the derivative of the density at the tabulated points of the VAL-atmosphere using a simple second order formula for nonequidistant points. Figure 1 shows the adiabatic sound speed and the derivative of the density as functions of the geometrical height. At the four lowest points of the VAL-atmosphere, the stratification is superadiabatic so that Eq. (70) yields complex frequencies. Also the HSRA-atmosphere (Gingerich et al. 1971) has this property, and even a density inversion. In the following we omit the first four points of the VAL-atmosphere.

For different wave numbers $k$, we now consider frequencies with certainly nonoscillatory solutions. Figure 2 shows boundary curves $v(z)=\omega(z) / 2 \pi$ defined by Eq. (70) for $k=0,1,2,2.485$, and 3 . In the range $2.485<k<7.6085$, where the regions are not connected, instead of the inequality (68) the inequalities (67) can be used. Therefore, the figures show the atmospheric $f$-mode with $\omega^{2}=g k$ and the so called "Lamb-mode" $\omega(z)=k c(z)$, denoted by $f$ and $L$. In the limit $k \rightarrow \infty$ we have:

$$
\sqrt{\frac{g}{c^{2}}\left(c^{2} \frac{\mathrm{d} \rho}{\mathrm{d} m}-g\right)}<\omega<c k
$$

This result is familiar: for the isothermal atmosphere, the square-root reduces to the constant Brunt-Vaisala-frequency. Between the Lamb-mode and the Brunt-Vaisala-frequency, waves are evanescent. As theorem 1 of Sect. 4 is only sufficient, the complementary regions of Fig. 2 may contain solutions with both oscillatory or nonoscillatory behavior.

\section{Conclusions}

Studies of the propagation behavior of linear adiabatic waves in empirical or theoretical model atmospheres usually comprise two steps: the numerical integration of the wave equation and the interpretation of the results. The first order Eqs. (1) and (2) are well suited for numerical integration. They are simple and the coefficients can be calculated without complications. However, to understand the results and get a deeper insight into the underlying physical effects, it is necessary to use a standard form of the wave equation. For standard forms, oscillation theorems and comparison theorems are powerful tools.

The invariant of the common standard form of the 3dimensional wave equation of plane atmospheres, given in Sect. 3 depends on second derivatives. This makes the wave equation too complicated for heuristic or interpretative studies. Even first derivatives of atmospheric quantities in the invariant can cause complications. It is for these reasons that we have sought suitable transformations that yield new standard forms of the wave equation with simple invariants. 

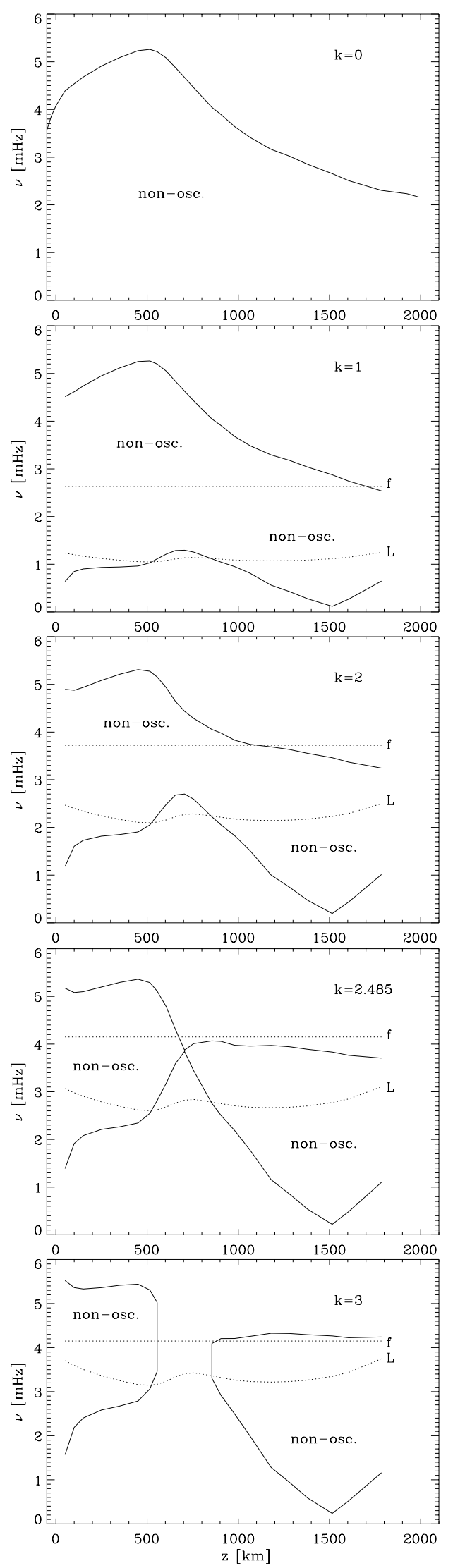

Fig. 2. The VAL-atmosphere: regions with certainly nonoscillatory waves. The solid curves limit the regions where Eq. (68) holds. The dotted curves are the f-mode and the "Lamb-mode" and limit the regions given by Eqs. (67).
The standard form (25) is still conventional. It offers the advantage that the independent variable, the logarithmic mass, is a very simple quantity. The invariant of this equation contains only the first derivative of the density, which makes this equation superior to previous standard forms of the wave equation.

In Sect. 5.2 we have adopted an unconventional approach and have replaced the wave equation by a non-linearly coupled system of two linear second order differential equations. The behavior of the invariants of these equations indicates an uncomplicated behavior of high frequency acoustic waves and of gravity waves. It is worth pointing out, that, for the latter, this includes the whole range $\omega^{2}<g k$ of gravity waves.

In Sect. 6, by some unconventional transformations, we were able to find a wave equation the invariant of which does not even contain the first derivative of the density. The disadvantage of this wave equation, however, is that the independent variable is no longer a function only of the geometrical height, but also a function of the parameter $\omega / k$. One thus has to discuss the invariant for a given $\omega / k$. We have therefore given some arguments for such a procedure which are related to Duvall's law. Like the wave equation given in Sect. 5.1, this wave equation, too, offers the advantage that, in the isothermal case, it reduces directly to the familiar wave equation of the isothermal atmosphere.

The invariant of the wave equation presented in Sect. 5.1 defines $z-\omega-k$ regions with certainly nonoscillatory solutions. For selected values of the horizontal wave number $k$ we have presented such regions in the $z-\omega$ plane.

A future paper will address specific features in the diagnostic diagram such as the p-mode ridges. We also hope that future studies in this area will clarify which of the two wave equations is the most effective.

\section{References}

Aizenman, M., Smeyers, P., \& Weigert, A. 1977, A\&A, 58, 41

Deubner, F.-L., \& Gough, D. O. 1984, ARA\&A, 22, 593

Duvall, T. L. Jr. 1982, Nature, 300, 242

Gingerich, O., Noyes, R. W., Kalkofen, W., \& Cuny, Y. 1971, Sol. Phys., 18, 347

Gradshteyn, I. S., \& Ryzhik, I. M. 1980, Tables of Integrals, Series, and Products (Academic Press, New York)

Lamb, H. 1932, Hydrodynamics (University Press, Cambridge)

Ledoux, P., \& Walraven, Th. 1958, in Handbuch der Physik, 51, ed. S. Flügge (Springer-Verlag, Berlin), 526

Leibacher, J. W., \& Stein, R. F. 1981, in the Sun as a Star, ed. J. Stuart, NASA SP-450, 263

Mathews, J., \& Walker, R. L. 1970, Mathematical Methods of Physics (W. A. Benjamin Inc., New York)

Mihalas, B. W., \& Toomre, J. 1981, ApJ, 249, 349

Schmitz, F., \& Fleck, B. 1994, A\&AS, 106, 129

Schmitz, F., \& Fleck, B. 1998, A\&A, 337, 487

Schmitz, F., \& Steffens, S. 1999, A\&A, 344, 973

Spruit, H. 1977, Thesis, University of Utrecht

Steffens, S. 1998, Thesis, University of Würzburg

Steffens, S., Deubner, F.-L., Hofmann, J., \& Fleck, B. 1995, A\&A, 302, 277

Steffens, S., \& Schmitz, F. 2000, A\&A, 354, 280

Steffens, S., Schmitz, F., \& Deubner, F.-L. 1997, Sol. Phys., 172, 85

Vernazza, J., Avrett, E., \& Loeser, R. 1981, ApJS, 45, 635

Wolf, B. E. 1983, A\&A, 127, 93 